\title{
Manejo del fuego como alternativa frente a los incendios forestales. El caso del Parque Entrenubes*
}

How to Manage the Flames as an Alternative in Cases of Forest Fires. The Case of Entrenubes Park

Marcos Edwin Quiroga Palacio ${ }^{a}$

Universidad de Ciencias Aplicadas y Ambientales de

Colombia, Colombia

rededwin@gmail.com

ORCID: http://orcid.org/0000-0002-9148-7891

Henny Margoth Santiago Villa

Universidad de Ciencias Aplicadas y Ambientales de

Colombia, Colombia

ORCID: http://orcid.org/0000-0003-4668-7533
DOI: https://doi.org/10.11144/Javeriana.ayd23-45.mfaf

Recepción: 13 Diciembre 2018

Aceptación: 20 Mayo 2019

Publicación: 30 Diciembre 2019

\section{Resumen:}

Es necesario entender el fuego como un fenómeno que hace parte de la dinámica de los socioecosistemas, debe tenerse una visión holística que va más allá de la simple supresión del mismo, forma actual de manejar los incendios forestales en Colombia. Este artículo hace un análisis del manejo del fuego para el Parque Ecológico Distrital de Montaña Entrenubes (Peden), en la ciudad de Bogotá D.C., orientado desde las Ciencias Ambientales y la ecología del fuego, donde se analizan las condiciones históricas y actuales de la incidencia de incendios en términos de número de incidentes, superficie afectada y distribución espacial; esto permite determinar la importancia de conocer el régimen actual de incendios para entender mejor el papel del fuego en este socioecosistema. Palabras clave: ecología del fuego, manejo del fuego, régimen de incendios forestales, camas combustibles, parques urbanos.

Abstract:

Today there is a need to understand the fire as phenomenon belonging to the dynamics of the social-ecosystems. The current way to manage a fire in Colombia also requires a holistic view beyond a mere removal of the fire. This article analyzes how the fire is being managed in the District Ecological Park of Montaña Entrenubes (Peden) in the city of Bogotá, D.C. This approach is based on the Environmental Sciences and the Ecology of Fire to analyze both the historic and current conditions of the fire incidence in terms of the numbers of events, affected surface and spatial distribution. It allows to determine how important is to know the current fire regulations to better understand the role of the fire in this social-ecosystem.

Keywords: fire ecology, fire management, forest fire regulations, combustible fuel beds, urban parks.

\section{Introducción}

En Colombia siempre se han manejado los incendios forestales desde una visión supresora del fuego, tanto así, que la legislación colombiana en el Decreto 146 de mayo de 2005, prohíbe cualquier actividad relacionada con el uso del fuego en zonas urbanas y rurales, a pesar de que en otros países se comenzó, desde hace años, la implementación del manejo del fuego basado en principios ecológicos (Florec et al., 2013; Jardel-Peláez, 2008, 2010, 2014; Jardel-Peláez, Castillo Navarro, Ramírez Villeda, Chacón Mathieu y Balcázar Medina, 2004; Jardel-Peláez et al., 2006; Jardel-Peláez, Alvarado, Morfín, Castillo y Flore, 2009; Jardel-Peláez et al., 2010; Lloret, 2004; Plana, 2004; Walker, Holling, Carpenter y Kinzig, 2004; Myers, Wade y Bergh, 2004; De Groot, Flannigan y Stocks, 2013; Pausas y Keeley, 2009; Santana, Jardel-Peláez y Pere, 2009; Hoeflich, Tetto y Batista, 2013).

Notas de autor

\footnotetext{
${ }^{a}$ Autor de correspondencia. Correo electrónico: rededwin@gmail.com
} 
La ecología estudia las interacciones entre el ambiente y los seres vivos, y actualmente considera los socioecosistemas como sistemas dinámicos y cambiantes, donde los eventos de perturbación hacen parte de su funcionamiento (Botkin, 1990; Terradas, 2001; Folke, 2006; Janssen y Ostrom, 2006). En la ecología moderna, el fuego ya no es considerado un factor externo que altera el equilibrio, sino un factor propio de los socioecosistemas que contribuye en su dinamismo; un fenómeno físico de la biosfera que consiste en un proceso de oxidación de la materia orgánica, en el cual una fuente de calor provoca el encendido y el inicio de la combustión (Pyne, Andrews y Laven, 1996).

Entender el manejo de los incendios forestales desde una mirada diferente a la de la supresión implica entrar en el campo de la interdisciplinariedad para analizar los regímenes históricos del fuego, las implicaciones del fuego en los procesos de la sucesión ecológica, el trabajo articulado con comunidades locales y combatientes del fuego, así como el análisis socioecológico de los espacios geográficos.

Esta investigación se realizó en el Parque Ecológico Distrital de Montaña Entrenubes, en la ciudad de Bogotá D.C. (Colombia), un socioecosistema (Janssen y Ostrom, 2006; Resilience Alliance, 2010; Morberg y Hauge Simonsen, 2016; Berkes y Folke, 1998; Walker et al., 2004) de gran valor para el Distrito Capital, por ser una reserva natural, un aula ambiental y por la importancia de las múltiples interacciones socioecológicas que en él se presentan. Este artículo forma parte del proyecto de investigación Implementación de estrategias de manejo del fuego basado en Principios Ecológicos. El caso del Parque Ecológico Distrital de Montaña Entrenubes, Bogotá D.C, ejecutado por el MSc Marcos Edwin Quiroga Palacio, en el año 2017.

El origen del parque se dio por un proceso de migración de comunidades rurales hacia la ciudad (Bello y Mosquera, 1999; Meertens, 1999; Tirado Mejía, 1997), las cuales conformaron y consolidaron barrios a su alrededor, en la década de los años ochenta; generaron presiones sobre sus bordes, mediante procesos de urbanización y actividades agrícolas, causas principales de los incendios forestales en la zona (Corporación Suna Hisca, 2003a, 2003b; POMA, 2003). Estos procesos de desplazamientos determinaron la transformación final de los relictos ecosistémicos en el interior del país (Márquez, 2001) y ocasionaron, a su vez, el crecimiento desorganizado de la ciudad, en donde no se ha logrado hacer una buena planificación para enfrentar las diferentes oleadas de desplazados que ha tenido que afrontar Bogotá durante los siglos XX y XXI.

Es fundamental entender que el fuego es un fenómeno que puede formar parte de la dinámica de un socioecosistema, más aún para el Parque Ecológico Distrital de Montaña Entrenubes, por poseer un marcado mosaico de vegetación, que presenta cobertura vegetal típica del bosque alto andino y subpáramo, acompañadas de especies con características de respuesta muy variables frente al fuego, entre estas, los bosques de pino (Pinus patula), bosques de eucalipto (Eucaliptus globulus), Ulex Europeaus, helechales y pastizales. Es por esto que se debe alcanzar una visión holística del fenómeno del fuego, para ir más allá de la simple supresión, siendo este el manejo actual de los incendios forestales en Colombia.

\section{Metodología}

Para dar cuenta de los objetivos del proyecto de investigación, se realizó una revisión de literatura que giró en torno a los ejes de incendios forestales y ecología del fuego. Esta información se logró en dos momentos. Mediante varias bases de datos como ProQuest, Science Direct y otras, y la documentación existente en las instituciones que hacen parte de la Comisión Distrital de Mitigación y Atención de Incendios Forestales (CDMAIF) de la ciudad de Bogotá D.C.; y durante la estancia en el Centro Universitario de la Costa Sur (CUCSUR), Universidad de Guadalajara (Autlán-Jalisco) en México, en el Doctorado en Ciencias en Biosistemática, Ecología y Manejo de Recursos Naturales y Agrícolas.

Se revisó la información estadística que se encuentra en las instituciones de la CDMAIF, para hacer un análisis sistémico desde el año 2010 de las condiciones históricas y actuales de la incidencia de incendios 
a través del tiempo, teniendo en cuenta: número de incidentes, superficie afectada y distribución espacial, así como la condición actual de los regímenes de incendios y los agentes de cambio en relación con dichos regímenes.

Esta información se analizó con la literatura sobre el tema, a la luz de las perspectivas de las Ciencias Ambientales, en donde la mirada interdisciplinar (McNeill, García-Godos y Gjerdåker, 2001; Flórez Malagón, 2002; Castro-Gómez, 2005; Max-Neef, 2005; Cubillos Quintero, 2007; González Ladrón de Guevara, 2007) se hace indispensable debido a la complejidad del problema (García, 1994; Vasco, 1995; Carrizosa, 2002; Leff, 2006, 2007), ya que permite un análisis más holístico de la situación, dado que los problemas o conflictos ambientales son complejos y requieren diversas miradas para su comprensión y solución.

Por otra parte, en la identificación del complejo de combustibles, se definieron diferentes coberturas vegetales de especial interés para estudios de manejo del fuego, debido a sus características de combustibilidad, relación con eventos de incendios, abundancia, y por generar ambientes de fuego diferentes; para esto, se tiene en cuenta la metodología planteada por Morfin-Ríos, Jardel Peláez y Michel Fuentes (2012), de clasificación de combustibles forestales por medio de fotoseries, que plantea dos fases.

Una de identificación general de las camas de combustibles, realizada durante los meses de julio a septiembre del 2016, y otra de evaluación cualitativa de las camas combustibles. Para esto se tuvieron en cuenta los tipos de vegetación más representativos del Parque Ecológico Distrital Entrenubes, a saber, vegetación típica del bosque alto andino, plantaciones de pino, plantaciones de eucalipto, parches de retamo espinoso, Ulex europaeus, Helechales de Pteridium aquilinum y pastizales de Pennisetum clandestinum. Todas estas analizadas en la segunda fase, mediante el método de evaluación cualitativa de las camas combustibles, basada en el enfoque y las variables consideradas en el Sistema de Clasificación de Características de Combustibles (FCCS, por sus siglas en inglés). Esta evaluación cualitativa permitió la identificación de grupos de camas combustibles.

\section{Resultados y Discusión}

El parque se ubica en la vertiente nororiental de la cuenca del río Tunjuelo, en la intersección de las localidades de San Cristóbal sur, Rafael Uribe y Usme; constituye junto con los cerros orientales las áreas protegidas más grandes de la ciudad de Bogotá D.C. Cuenta con 626,4 ha, fraccionado en tres formaciones: cerro Guacamayas (40,21 ha), cerro Juan Rey (402,57 ha) y la cuchilla del Gavilán (183,61 ha). Esta zona ha tenido múltiples procesos de urbanización informal a través del tiempo, que ha llevado a generar transformaciones socioecosistémicas dentro de la ciudad (Corporación Suna Hisca, 2003a, 2003b; POMA, 2003).

En la identificación del complejo de combustibles en el parque, se definieron diferentes coberturas vegetales de especial interés para estudios de manejo del fuego, como plantaciones de pino (Pinus patula, Pinus radiata), de eucalipto (Eucaliptus globulus), pastizales (Pennisetum clandestinum), matorrales de retamo espinoso (Ulex europaeus), helechales (Pteridium aquilinum) y vegetación nativa típica del bosque alto andino. El 50\% de estas coberturas vegetales son foráneas (pino, eucalipto, retamo espinoso y pastizales) y tienen atributos para la generación de incendios de severidad baja, mixta o alta (Jardel-Peláez, 2014; Skolmen y Ledig, 1990; Tapias, Bertomeu, Gil y Pardos, 1997). Estas especies poseen alta capacidad de reproducción y de persistencia ante perturbaciones, especialmente fuego y tala, además de una alta competitividad sobre las especies nativas, y presentan grandes atributos pirogénicos: para el caso de Bogotá, el retamo espinoso se ha convertido en un grave problema a la hora de su erradicación.

En este ecosistema, las plantaciones de pino están principalmente representadas por la especie Pinus patula, la cual posee atributos de adaptación al fuego, entre los que se encuentran la resistencia a incendios superficiales de baja intensidad y cortezas resistentes que protegen el cambium de las altas 
temperaturas (Rodríguez-Trejo y Fulé, 2003). Así mismo, las plantaciones de eucalipto de la especie Eucaliptus globulusposeen atributos que los relacionan con la presencia de incendios periódicos en Australia, como son las resinas altamente inflamables y la resistencia de sus fustes debido a una prominente corteza (Ashton, 1981).

En el caso de los pastizales, representados por Pennisetum clandestinum y los helechales Pteridium aquilinum, estos son capaces de generar camas combustibles altamente inflamables, debido a la rápida regeneración de las especies y la acumulación de material vegetal en periodos secos (Jardel-Peláez, 2014; Crane, 1990). Muchas especies de gramíneas toleran o incluso potencian el fuego, y muchas responden al fuego con rápido crecimiento (D’Antonio y Peter, 1992). Los helechales de Pteridium aquilinum por su parte, no solo están bien adaptados al fuego, sino que promueven ambientes propensos al fuego por la producción de biomasa muy seca e inflamable (Agee y Huff, 1987).

Finalmente, la capacidad de recuperación de la vegetación nativa típica del bosque alto andino, conformada por especies vegetales sensibles a los incendios, como por ejemplo, Encenillo Weinmannia tomentosa, Corono Xylosmaspiculifera, Raque Vallea stipularis, Chusque Chusquea scandens, Palo blanco Ilexkunthiana, Arrayán Myrcianthes leucoxyla, Aliso Alnus acuminata, Tuno Miconia squamulosa, entre otros, ese ve afectada por especies pioneras como los pastos y arbustos del páramo y subpáramo, que ocupan ágilmente los terrenos donde el bosque altoandino ha sido eliminado (Departamento Técnico Administrativo Medio Ambiente [DAMA ], 2000). Además, se trata de bosques que típicamente se entienden como independientes del fuego y, siendo este tipo de vegetación una de las más representativas del territorio colombiano, se tiende a generalizar en Colombia la idea del fuego ausente en la vegetación (Hardesty, Myers y Fulks, 2005).

Es así que el panorama de camas combustibles, para el ecosistema Parque Ecológico Distrital de Montaña Entrenubes, convierte en un verdadero reto el manejo de este e invita a la necesidad de entender y transitar en el manejo del fuego. El establecimiento de especies exóticas que conservan sus adaptaciones y predisposiciones a los incendios forestales (Rodríguez-Trejo, 2014), conviviendo en el mismo sistema ecológico de aquellas especies nativas que no poseen los mismos atributos frente al fuego, dan como resultado una combinación de combustibles forestales e inflamabilidad de las especies, que puede derivar en diferentes efectos y severidades del fuego, haciendo de este ecosistema un lugar altamente vulnerable frente a los incendios forestales. Sumado a esto, actividades antrópicas que se convierten en un gran detonante de los incendios, como la quema de basuras urbanas de los barrios aledaños al parque y en general comunidades que están generando presión antrópica constante sobre este.

El concepto de incendio forestal cuenta con múltiples y variadas definiciones, así como puntos de vista: cada región lo define de acuerdo con las características propias de la cobertura vegetal que posee su geografía. En Colombia, el Sistema de Información Ambiental de Colombia (SIAC) define el incendio sobre la cobertura vegetal como el fuego que se expande sin control y sin límites preestablecidos, consumiendo material vegetal ubicado en áreas donde predominan los bosques o en aquellas que sin serlo tengan importancia ambiental (Vélez, 2000).

En la historia de la humanidad, el fuego ha hecho parte del proceso natural, social y cultural del planeta y especialmente de la especie humana desde su proceso evolutivo, produciendo fuertes consecuencias ecológicas y evolutivas para cualquier tipo de vida (Pausas y Keeley, 2009). Es fundamental conocer la incidencia de incendios a través del tiempo, la condición actual de los regímenes de incendios y los agentes de cambio en relación con los regímenes históricos, para concebir mejor el fuego y el papel ecológico que este ha desempeñado a lo largo del tiempo. Así mismo, para la determinación del manejo del fuego en una unidad de paisaje es necesario conocer la condición actual del régimen de incendios y el régimen potencial de incendios.

Conocer los regímenes de incendios forestales es necesario para establecer un adecuado manejo del fuego, con esta información se define como un socioecosistema específico o una unidad de manejo determinada evoluciona y se adapta naturalmente al fuego, obteniendo así nociones del papel ecológico que el fuego desempeña en este y su cobertura vegetal. El régimen de incendios forestales se puede definir como la 
recurrencia, la intensidad y el momento (estacionalidad) de los incendios, donde las condiciones son determinadas por las características del clima y de la vegetación. Sin embargo, los efectos antrópicos negativos sobre los ecosistemas, se reflejan en alteraciones de los regímenes de incendios, en algunos casos, incrementando el número de incendios, en otros, suprimiéndolos casi en su totalidad (Lloret, 2004).

La precipitación anual reportada por el Instituto de Hidrología, Meteorología y Estudios Ambientales (Ideam) evidencia que el año 2015 tuvo la menor precipitación anual de los últimos 35 años. La humedad de los combustibles es considerada como el factor más significativo y variable que determina su inflamabilidad (Morfin-Ríos et al., 2012), en este caso la precipitación considerablemente baja del año 2015 reduce la humedad de los combustibles, siendo estos más propensos a la ignición. Dentro del contexto histórico, este año registra el menor volumen de precipitación anual del país de los últimos 35 años, con una reducción del $23 \%$ en el acumulado anual. Del mismo modo, la temperatura anual del aire reportada por el Ideam muestra que el 2015 ha sido el año más cálido de los últimos 35 años, con una anomalía de $0,85^{\circ} \mathrm{C}$ por encima de los valores promedios respecto al periodo 1981-2010 (Ideam, Grupo de Climatología y Agroclimatología y Ministerio de Ambiente, 2015).

Estos reportes de precipitación y variación en la temperatura nos indican la presencia de condiciones meteorológicas extremas (baja humedad, altas temperaturas), donde los incendios tienden a ser más intensos y propagarse en áreas más extensas (Jardel-Peláez et al., 2010). Para el caso del Peden, estas condiciones meteorológicas están estrechamente relacionadas con la ocurrencia de los incendios forestales, donde el año 2015 muestra el mayor número de incidentes de acuerdo con los reportes de la CDMPIF (figura 1). Los reportes oficiales de estos incendios forestales los catalogan como provocados por dinámicas antrópicas.

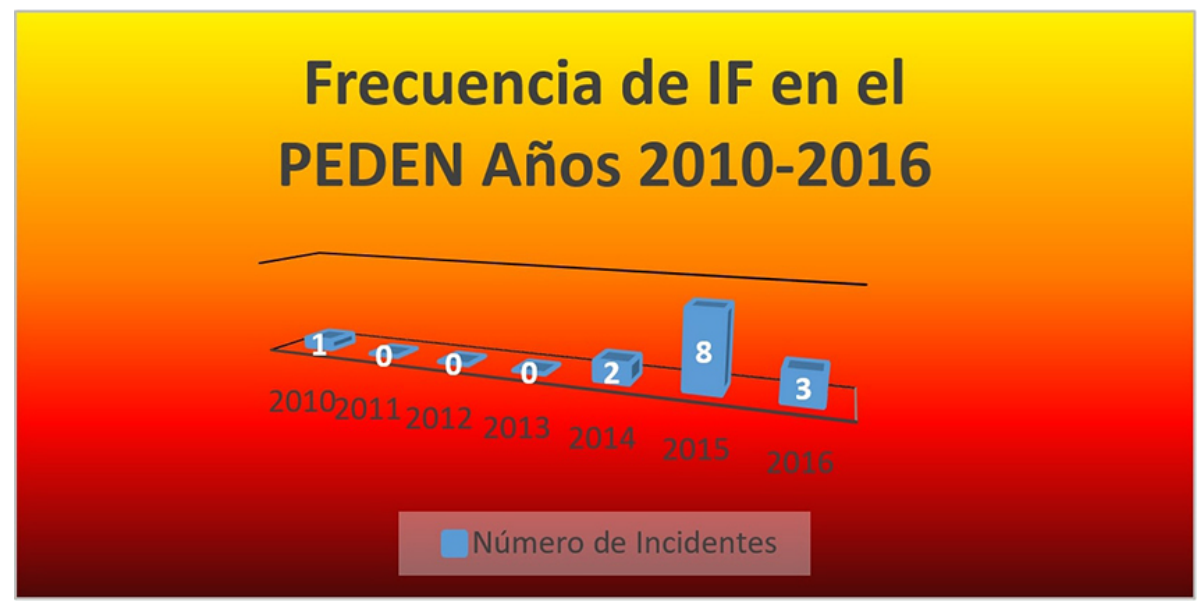

FIGURA 1.

Frecuencia de incendios forestales en el Peden, años 2010-2016

Fuente: elaboración propia

En cuanto a la condición actual del régimen de incendios forestales, el cual consiste en el régimen de incendios observado en los últimos años y que puede ser, en algunos casos, semejante al régimen histórico o al régimen potencial (Jardel-Peláez, 2010), para el Parque Ecológico Distrital de Montaña Entrenubes se reportan incendios desde el 2010, en las estadísticas oficiales de la CDMPIF. Para el análisis se tienen en cuenta características de régimen de incendios como la frecuencia, la intensidad y la estacionalidad. y los reportes de incendios, quemas y conatos; estos conceptos difieren estadísticamente a razón del área afectada, sin embargo, se tienen en cuenta todos, puesto que la atención de estas emergencias en el Distrito Capital es inmediata, lo cual reduce considerablemente el área total afectada. En la figura 1 se muestra la frecuencia de incendios forestales que se refiere al número de incendios por unidad de tiempo en el parque.

De otra parte, se exploró información documental en el periódico El Tiempo, desde 1950 hasta la fecha, dando resultados negativos. La ausencia de esta información impidió reconstruir el régimen histórico de 
incendios forestales de forma documental, por lo que se recomienda hacer análisis dendrocronológicos de cicatrices de fuego, con el fin de generar una reconstrucción histórica y la descripción de las características inmediatas del régimen del fuego que ha afectado al socioecosistema en mención (Medina, 2007).

La estacionalidad hace referencia a las temporadas del año en que se presentan los incendios forestales, donde las condiciones meteorológicas de sequía aumentan la probabilidad de la propagación de incendios; la estación de incendios varía en duración (número de días) y época del año en diferentes tipos de clima (JardelPeláez, 2010; Tapias et al., 1997). Para el caso de estudio, la duración típica de la época seca comprende los meses diciembre-marzo y julio-septiembre. Así, teniendo en cuenta los incendios reportados oficialmente en los años 2010 a 2016, se muestra una mayor ocurrencia durante el mes de enero, con un total de incidentes que representan el $38 \%$ de los reportes (figura 2).

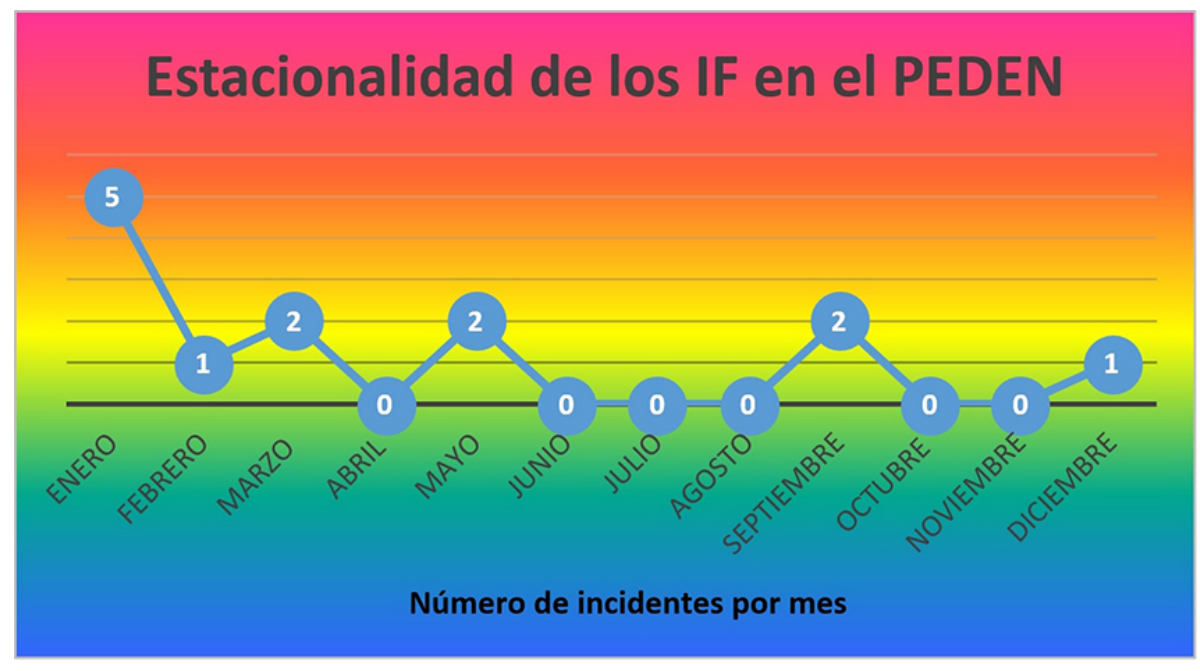

FIGURA 2.

Estacionalidad de los IF presentados en el Peden, según reportes oficiales 2010-2016 Fuente: elaboración propia

También, de acuerdo con los datos obtenidos de los reportes, se pudo determinar la superficie afectada por IF en el periodo 2010-2016 (figura 3), siendo el año 2014 el que mostró una mayor afectación, con un total de 5 ha. Esto está posiblemente relacionado con la ubicación de los eventos y la accesibilidad de los cuerpos de bomberos que, en algunas ocasiones por las dificultades de caminos, tardan en la primera atención para el combate de incendios, aumentado así las probabilidades de avance del fuego. Además, las prácticas antrópicas identificadas en los barrios vecinos agudizan la situación, dado que las quemas realizadas por las comunidades son más propensas a salirse de control en temporadas de estacionalidad seca. 


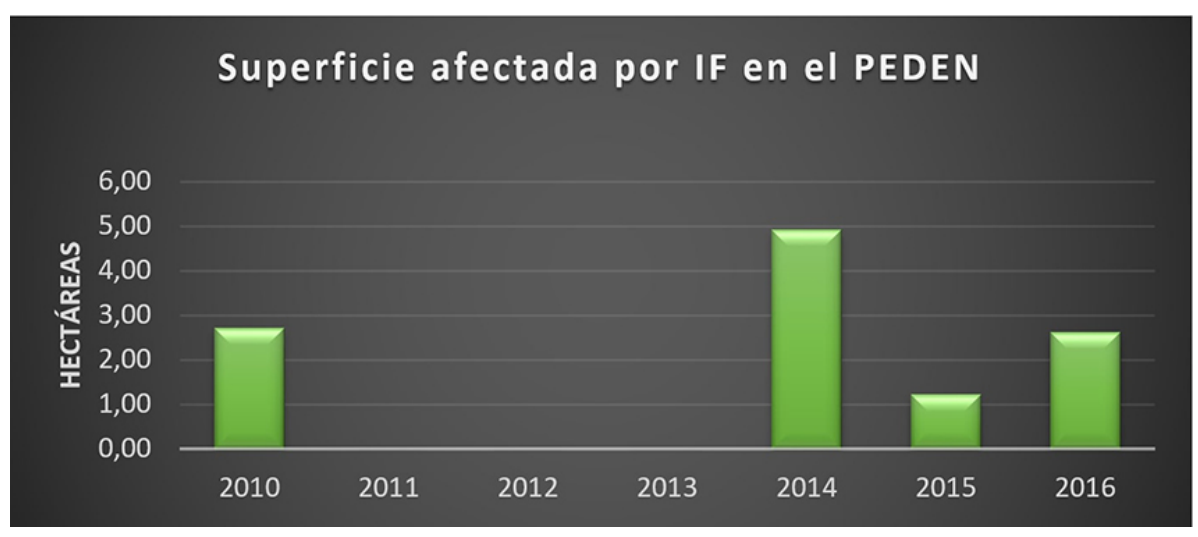

FIGURA 3.

Superficie afectada por IF en el Peden, según reportes oficiales 2010-2016 Fuente: elaboración propia

La ocurrencia de incendios reportados en el parque concuerda con la estacionalidad de Bogotá y del occidente del departamento de Cundinamarca, caracterizada por el régimen bimodal, propio de los Andes, donde se alternan períodos de lluvias con períodos secos; el primer periodo de lluvias va de abril a mayo, se alterna con un periodo seco entre julio a septiembre, y el segundo periodo de lluvias se presenta de octubre a noviembre, con un periodo seco de diciembre a febrero (Ideam, 2014). Éste comportamiento estacional se evidencia en algunas de las estaciones meteorológicas presentes en la cuenca de influencia del parque (Corporación Suna Hisca, 2003a). Sin embargo, cuando los fenómenos de El Niño y La Niña se presentan, estas tendencias estacionales se vuelven difusas a lo largo del año, presentándose lluvias o sequías durante más meses de los acostumbrados (Ideam, 2014).

El fenómeno de El Niño en la región del Distrito Capital se expresa por un aumento de la temperatura media de hasta $0,5^{\circ} \mathrm{C}$ y disminución de lluvias que llega a ser en algunos sectores hasta del $60 \%$ (Ideam, 2014). Esta condición puede generar sequias que favorecen la ocurrencia de incendios forestales, sobre todo en este tipo de socioecosistemas, con presencia de especies pirogénicas exóticas como el retamo espinoso (Ulex europeaus) y el pino (Pinuspatula, Pinus radiata), y de especies nativas como el helecho marranero (Pteridium aquilinum) y el laurel hojipequeño (Morella parvifolia).

La variabilidad climática que se presenta por causa de los fenómenos de El Niño y de La Niña se debe tener en cuenta para la planificación del manejo del fuego, ya que estos fenómenos incrementan el riesgo de desastres en el territorio. Durante el fenómeno de El Niño, predominan los eventos de incendios forestales y deslizamientos por sequedad de los terrenos. El Niño de los años 2015-2016 se ubica, junto a los de los años 1997-1998, 1982-1983, entre los más fuertes desde 1950 y su evolución actual es inédita (Centro Internacional para la Investigación del Fenómeno de El Niño [Ciifen], 2017). Estos datos de probabilidad climática debida al fenómeno de El Niño están fuertemente relacionados con la realidad vivida en Bogotá durante los meses de diciembre 2015, enero, febrero, marzo y abril de 2016, debido que se multiplicaron las emergencias por incendios forestales en la capital, afectando alrededor de 150 ha en el primer trimestre del año 2016.

De acuerdo a lo anterior, la condición actual del régimen de incendios forestales en el parque, en cuanto a frecuencia, intensidad y estacionalidad, evidencia una correlación marcada con las condiciones climatológicas características de la ciudad y se ve influenciada por las condiciones climáticas dadas durante el fenómeno de El Niño, principalmente. Además, la superficie afectada se ve relacionada también con variables sociales, principalmente por las condiciones generadas por las comunidades aledañas y la labor de los organismos de respuesta a estos eventos.

Todo lo anterior lleva a analizar la necesidad de generar una propuesta de manejo del fuego; para tal fin, en cualquier socioecosistema, se debe tener en cuenta el comportamiento potencial del fuego, representado 
por el triángulo del ambiente del fuego, conformado por el tiempo atmosférico, la topografía y el combustible (Morfin-Ríos et al., 2012). Este comportamiento del fuego sirve para identificar el peligro de incendios y da cuenta de la severidad potencial y la resistencia al control, siendo las propiedades físicas de los combustibles las únicas manipulables como método de reducción del riesgo.

Como se ha mencionado, la principal causa de incendios forestales en el parque son las prácticas de origen antrópico, a pesar de la vigilancia permanente con que se cuenta, no todo el territorio queda protegido. La información dada por la administración muestra que la vigilancia se ubica en las zonas con mayor incidencia de incendios forestales, especialmente en los bordes del parque, que reciben la presión de las comunidades que limitan con él. A partir de la información relacionada con las causas de los incendios forestales y los puntos de mayor ocurrencia de incendios, se estableció una zonificación de riesgo de incendio para el parque, como se ve en la figura 4.

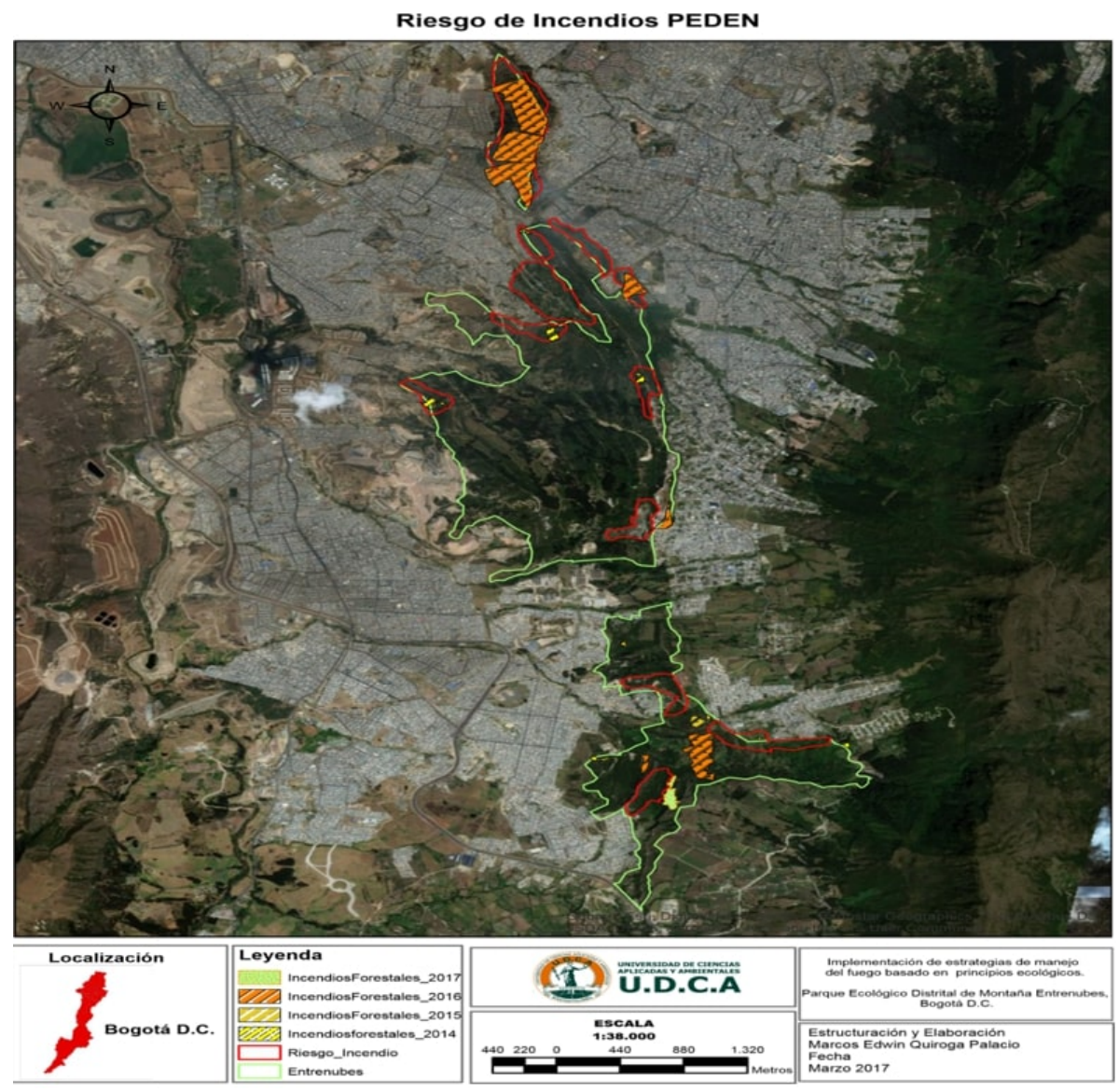

FIGURA 4.

Mapa de riesgos por incendios forestales en el Parque Ecológico de Montaña Entrenubes Fuente: Quiroga Palacio (2017)

El programa de manejo del fuego en este parque debe orientarse teniendo en cuenta que es un área de conservación ecológica y un aula ambiental, y debe contar con una descripción de las características y condiciones socioecológicas de este, que articule procesos de comprensión sobre la dinámica del fuego en las diferentes coberturas vegetales, el papel ecológico que este pueda desempeñar en ellas, la prohibición del uso del fuego y las campañas de prevención.

Se determina que la total supresión del fuego en este socioecosistema no es la respuesta de manejo ideal por la complejidad del socioecosistema, por lo tanto, la manera más adecuada de abordarlo es la tendencia multienfoque, es decir, usar el fuego como herramienta de gestión del riesgo en las zonas que presentan peligro de incendio para controlar la acumulación de cargas de combustibles, la supresión total del fuego en aquellas 
coberturas vegetales sensibles al fuego, un verdadero control de las prácticas generadoras de incendios en las zonas demarcadas como riesgo de incendio, y un mejorado sistema de comunicación y acceso para los cuerpos de bomberos en caso de presentarse la emergencia.

Este tipo de manejo del fuego que se propone es denominado manejo mixto, donde se debe entender muy bien el papel del fuego en el lugar de estudio, determinando así la importancia ecológica del fuego y los factores negativos de este en las diferentes zonas del mismo socioecosistema.

\section{Conclusiones}

En este trabajo se analizan las condiciones históricas y actuales de la incidencia de incendios, en términos de número de incidentes, superficie afectada y distribución espacial; esto permite determinar la importancia de conocer el régimen actual de incendios en el Parque Entrenubes, con el fin de entender mejor el papel del fuego en el socioecosistema, apoyado desde la ecología del fuego.

Los incendios forestales en el parque Entrenubes, a pesar de ser causados en su gran mayoría por actividades antrópicas, tienen una directa relación con las condiciones atmosféricas de estacionalidad y con la vegetación que se ha determinado como más propensa a los incendios, como pastos, bosques de pino, eucalipto y parches de retamo espinoso.

Con esto, se entiende que el manejo del fuego no debe estar basado únicamente en la prohibición del uso del fuego y en campañas de prevención, este manejo debe entender la dinámica del fuego sobre las diferentes coberturas vegetales y el papel ecológico que este desempeña, así se podrán establecer estrategias de manejo de fuego ideales para los socioecosistemas, aspecto que va avanzado en países como México, Chile, Estados Unidos y Australia, y que en Colombia es necesario implementar.

El manejo del fuego, basado en principios ecológicos, es un proceso de educación y gestión ambiental que debe articular diferentes conocimientos y actores sociales: instituciones, comunidades locales, combatientes del fuego; generando así, mejores resultados en los procesos de prevención y mitigación. Con lo determinado en esta investigación, se propone como estrategia para el manejo del fuego en el Peden un enfoque mixto donde se entienda el papel ecológico del fuego y su importancia en diferentes coberturas vegetales adaptadas al fuego, la supresión en algunas coberturas vegetales independientes del fuego, el trabajo comunitario para comprender el papel de la comunidad en la ocurrencia del fenómeno, y el mejoramiento de los sistemas de atención y respuesta a las emergencias por fuego en los bosques.

\section{Referencias}

Agee, J. K., y Huff, M. H. (1987). Fuel succession in a western hemlock/Douglas-fir forest. Canadian Journal of Forest Research, 17(7), 697-704. https://doi.org/10.1139/x87-112

Ashton, D. H. (1981). Fire in tall open-forests (wet sclerophyll forests). En A. M. Gill, R. H. Groves e I. R. Noble (eds.), Fire and the Australian biota. Canberra City, ACT: The Australian Academy of Science (pp. 339-366). Canberra: Australian Academy of Science.

Bello, M. N., y Mosquera, C. (1999). Desplazados, migrantes y excluidos: actores de las dinámicas urbanas En F. Cubides y C. Domínguez (eds.), Desplazados, migraciones internasy reestructuracionesterritoriales (pp. 456-474). Santafé de Bogotá: Observatorio Sociopolítico y Cultural, Centro de Estudios Sociales, Universidad Nacional de Colombia.

Berkes, F., y Folke, C. (1998). Linking social and ecological systems for resilience and sustainability. En F. Berkes y C. Folke (eds.), Linking social and ecological system management practices and social mechanisms for building resilience (pp. 1-26). Cambridge: Cambridge University Press.

Botkin, D. B. (1990). Discordant Harmonies. Nueva York: Oxford University Press. 
Carrizosa, J. (2002). Colombia de lo imaginario a lo complejo. Bogotá: IDEA. Universidad Nacional.

Castro-Gómez, S. (2005). Transdisciplinariedad y diálogo de saberes. En Miradas y Perspectivas de las Ciencias Sociales y Humanas. Bogotá: Instituto Pensar. EDES. Universidad Javeriana.

Centro Internacional para la Investigación del Fenómeno de El Niño (Ciifen). (2017). El Niño 2015-16: evolución, vulnerabilidad e impactos en Latinoamérica. Guayaquil, Ecuador. Recuperado de http://www.ciifen.org/

Corporación Suna Hisca. (2003a). Parque Ecológico Distrital de Montaña Entrenubes. Tomo I. Componente Biofisico. Bogotá: Departamento Técnico Administrativo del Medio Ambiente.

Corporación Suna Hisca. (2003b). Parque Ecológico Distrital de Montaña Entrenubes. Tomo II. Componente Social. Bogotá: Departamento Técnico Administrativo del Medio Ambiente.

Crane, M. F. (1990). Species: Pteridium aquilinum. Fire Effects Information System. U.S. Department of Agriculture, Forest Service, Rocky Mountain Research Station, Fire Sciences Laboratory. Recuperado de http://www.fs.fed .us/database/feis/

Cubillos Quintero, L. F. (2007). La epistemología de las ciencias ambientales. Reflexiones desde la "impertinencia" social. En O. Sáenz (comp.), Las Ciencias Ambientales: Una nueva área de conocimiento (pp. 83-92). Bogotá: RCFA.

D’Antonio, C., y Peter, M. (1992). Biological Invasions by Exotic Grasses, the Grass/Fire Cycle, and Global Change. Annual Review of Ecology and Systematics, 23, 63-87. https://doi.org/10.1146/annurev.es.23.110192.000431

De Groot, W. J., Flannigan, M. D., y Stocks, B. J. (2013). El Cambio Climático y los Incendios Forestales. En A. González-Cabán (coord.), Memorias del Cuarto Simposio Internacional Sobre Políticas, Planificación y Economia de los Incendios Forestales: Cambio Climático e Incendios Forestales (pp. 1-12). Ciudad de México: Departamento de Agricultura de los EE.UU., Servicio Forestal, Estación de Investigación del Pacífico Suroeste.

Decreto 146 de mayo de 2005. Creación de la Comisión Distrital para la Prevención y Mitigación de Incendios Forestales. Alcaldía Mayor de Bogotá. Recuperado de http://ambientebogota.gov.co/c/document_library/get_ file?uuid=dd3f4ac9-b33c-4b37-ba5d-c44b658d081e\&groupId $=10157$

Departamento Técnico Administrativo Medio Ambiente (DAMA). (2000). Protocolo Distrital de Restauración Ecológica. Guía para la restauración de ecosistemas nativos en las áreas rurales de Santa Fe de Bogotá. Alcaldía Mayor de Santafé de Bogotá.

Florec, V. Pannell, D., Burton, M., Kelso, J., Mellor, D., y Milne, G. (2013). Análisis Económico de la quema prescrita para la gestión de Incendios en Australia Occidental. En A. González-Cabán (coord.), Memorias del Cuarto Simposio Internacional Sobre Politicas, Planificación y Economia de los Incendios Forestales: Cambio Climático e Incendios Forestales (pp. 110-123). Ciudad de México: Departamento de Agricultura de los EE.UU., Servicio Forestal, Estación de Investigación del Pacífico Suroeste.

Flórez Malagón, A. G. (2002). Disciplinas, transdisciplinas y el dilema holístico: una reflexión desde Latinoamérica. En A.G. Flórez Malagón y C. Millán de Benavides (eds.), Desafios de la transdiciplinariedad (pp. 128-155). Bogotá: Instituto Pensar, Pontificia Universidad Javeriana.

Folke, C. (2006). Resilience: the emergence of a perspective for social-ecological system analysis. Global Environmental Change, 16(3), 253-267. https://doi.org/10.1016/j.gloenvcha.2006.04.002

García, R. (1994). Interdisciplinariedad y sistemas complejos. En E. Leff (ed.), Ciencias Sociales y formación ambiental (pp. 85-124). Madrid: Editorial Gedisa.

González Ladrón de Guevara, F. (2007). Aportes para una caracterización de las ciencias ambientales. En O. Sáenz (comp.), Las Ciencias Ambientales: Una nueva área de conocimiento (pp. 35-48). Bogotá: RCFA.

Hardesty, J., Myers, R. L., y Fulks, W. (2005). Fire, ecosystems, and people: a preliminary assessment of fire as a global conservation issue. The George Wright Forum, 22(4), 78-87. http://www.georgewright.org/224hardesty.pdf

Hoeflich, V. A., Tetto, A. F., y Batista, A. C. (2013). Información económica sobre el comportamiento histórico de los Incendios Forestales en los Bosques en el Estado de Paraná, Brasil. En A. González-Cabán (coord.), Memorias del Cuarto Simposio Internacional Sobre Politicas, Planificación y Economia de los Incendios Forestales: Cambio Climático e Incendios Forestales (pp. 142-154). Ciudad de México: Departamento de Agricultura de los EE.UU., Servicio Forestal, Estación de Investigación del Pacífico Suroeste. 
Instituto de Hidrología, Meteorología y Estudios Ambientales (Ideam). (2014). Evolución de precipitación y temperatura durante los fenómenos El Niño y La Niña en Bogotá - Cundinamarca (1951 - 2012). Elementospara la acción institucional. Bogotá, Colombia: IDEAM, PNUD.

Instituto de Hidrología, Meteorología y Estudios Ambientales (Ideam), Grupo de Climatología y Agroclimatología y Ministerio de Ambiente. (2015). Anuario Climatológico. Bogotá, Colombia. Recuperado de http://www.ideam.gov.co/documents/21021/299660/Anuario_Climatologico_2015.pdf/ea469095-34a1442f-bfb4-741f0df69c32?version $=1.0$

Janssen, M. A., y Ostrom, E. (2006). Governing social-ecological systems, Capítulo 30. Handbook of Computational Economics, 2, 1465-1509. https://doi.org/10.1016/S1574-0021(05)02030-7

Jardel-Peláez, E. J. (2008). Sucesión ecológica y restauración de bosques subtropicales de montaña en la Estación Científica Las Joyas, México. En M. González-Espinosa, J. M. Rey-Benayas, N. Ramírez-Marcial (eds.), Restauración de Bosques en América Latina (pp. 77-97). México D. F.: Mundi-Prensa/ Fundación Internacional para la Restauración de Ecosistemas.

Jardel-Peláez, E. J. (2010). Planificación del Manejo del Fuego. Autlán, Jalisco: Universidad de Guadalajara - Fundación Manantlán para la Biodiversidad de Occidente-Consejo Civil Mexicano para la Silvicultura Sostenible-Fondo Mexicano para la Conservación de la Naturaleza.

Jardel-Peláez, E. J. (2014). Plan de manejo del fuego de la reserva de la biosfera Trifinio-Fraternidad República el Salvador. San Salvador: Ministerio de medio ambiente y recursos naturales. Programa del Hombre y la Biosfera, Organización de las Naciones Unidas para la Educación, la Ciencia y la Cultura.

Jardel-Peláez, E. J., Alvarado, J. E., Morfín R., F., Castillo, N., y. Flore, J. G. (2009). Regímenes de fuego en ecosistemas forestales de México. En G. Flores (coord.), Impacto ambiental de incendios forestales (pp. 73-100). México D. F.: Mundi Prensa México.

Jardel-Peláez, E. J., Castillo Navarro, F., Ramírez Villeda, R., Chacón Mathieu, C., y Balcázar Medina, O. (2004). Los incendios forestales en la Reserva de la Biosfera Sierra de Manantlan, Jalisco-Colima. En R. Villers, y J. LópezBlanco (eds.), Incendios forestales en México (pp. 143-160). México D. F.: Centro de Ciencias de la Atmósfera, UNAM.

Jardel-Peláez, E. J., Frausto Leyva, J. M., Pérez Salicrup, D., Alvarado Celestino, E., Morfín Ríos, J. E., Landa, R., y Llamas Casillas, P. (2010). Prioridades de investigación en manejo del fuego en México. México D. F.: Fondo Mexicano para la Conservación de la Naturaleza.

Jardel-Peláez, E. J., Ramírez-Villeda, R., Castillo-Navarro, F., García-Ruvalcaba, S., Balcázar, O., Chacón Mathieu, J. y Morfin Ríos, J. (2006). Manejo del Fuego y Restauración de Bosques en la Reserva de la Biosfera Sierra de Manantlán, México. En J. G. Flores-Garnica, y D. Rodrígez-Trejo (eds.), Incendios Forestales (pp. 1-22). México y Madrid: Mundi-Prensa-CONAFOR.

Leff, E. (2006). Complejidad, racionalidad ambiental y diálogo de saberes. En Congreso internacional interdisciplinar de participación, animación e intervención socioeducativa (pp. 1-12). Barcelona: Centro Nacional de Educación Ambiental.

Leff, E. (2007). La complejidad ambiental: del logos científico al diálogo de saberes. En O. Sáenz (comp.), Las Ciencias Ambientales: Una nueva área de conocimiento (pp. 53-62). Bogotá: RCFA.

Lloret, F. (2004). Régimen de incendios y regeneración. En F. Valladares (ed.), Ecología del bosque mediterráneo en un mundo cambiante (pp. 101-126). Madrid: Ministerio de Medio Ambiente, EGRAF S.A.

Márquez, G. (2001). De la abundancia a la escasez: la transformación de ecosistemas en Colombia. En G. Palacio (ed.), Naturaleza en disputa. Ensayos de Historia Ambiental de Colombia 1850-1995 (pp. 321-480). Bogotá: Universidad Nacional de Colombia.

Max-Neef, M. (2005). Foundation of transdisciplinarity. Ecological Economics, 53(1), 5-16. https://doi.org/10.1016/ j.ecolecon.2005.01.014

McNeill, D., García-Godos J., y Gjerdåker, A. (eds.). (2001). Interdisciplinary Research on Development and the Environment. Oslo: Centre for Development and the Environment University of Oslo. 
Medina, A. (2007). Reconstrucción de los regímenes de fuego en un bosque de Prosopiscaldenia, provincia de La Pampa, Argentina. Bosque (Valdivia), 28(3),234-240. http://dx.doi.org/10.4067/S0717-92002007000300008

Meertens, D. (1999). Desplazamiento interno y género: trayectorias y estrategias de reconstrucción vital. En F. Cubides y C. Domínguez (eds.), Desplazados, migraciones internas y reestructuraciones territoriales (pp. 406-454). Santafé de Bogotá: Observatorio Sociopolítico y Cultural, Centro de Estudios Sociales, Universidad Nacional de Colombia.

Morberg, F., y Hauge Simonsen, S. (2016). ¿Qué es la resiliencia? Una introducción a la investigación sobre el sistema socio-ecológico. Estocolmo: Stockolm Resilience Centre.

Morfin-Ríos, J. E., Jardel Peláez, E. J., y Michel Fuentes, J. M. (2012). Caracterización y cuantificación de combustibles forestales. Guadalajara, Jalisco: Comisión Nacional Forestal-Universidad de Guadalajara, Editorial Universitaria.

Myers, R., Wade, D., y Bergh, C. (2004). Fire Management Assessment of the Caribbean Pine (Pinus caribea) Forest Ecosystems on Andros and Abaco Islands, Bahamas. GFI publication No. 2004-1. Arlington: The Nature Conservancy.

Pausas, J. G., y Keeley, J. E. (2009). Burning Story: The Role of Fire in the History of Life. BioScience, 59(7), 593-601. http://dx.doi.org/10.1525/bio.2009.59.7.10

Plana, E. (2004). Anàlisi d'escenaris de prevenció i extinció d'incendis des de la perspectiva socioambiental. En E. Plana (coord.), Incendis forestals, dimensió socioambiental, gestió del risc i ecologia del foc (pp. 5-12). Solsona, Cataluña: Xarxa Temàtica de Recerca Alinfo.

POMA. (2003). Parque Ecológico Distrital de Montaña Entrenubes. Plan de Ordenamiento y Manejo POMA. Bogotá: Departamento Técnico Administrativo del Medio Ambiente.

Pyne, S. J., Andrews, P. L., y Laven, R. D. (1996). Introduction to wildland fire. Nueva York: John Wiley.

Quiroga Palacio, E. (2017). Estrategias de manejo del fuego basado en principios ecológicos. El caso del Parque Ecológico Distrital de Montaña Entrenubes, Bogotá D.C. Recuperado de https://repository.udca.edu.co/handle/11158/6 98

Resilience Alliance. (2010). Assessing resilience in social-ecological systems: workbook for practitioners. Version 2.0. Recuperado de https://www.resalliance.org/3871.php

Rodríguez-Trejo, D. A. (2014). Incendios de Vegetación. Su Ecología, Manejo e historia (Vol. 1). México: C. P., UACH, Semarnat, Conafor, Conanp.

Rodríguez-Trejo, D. A., y Fulé, P. Z. (2003). Fire ecology of Mexican pines and a fire management proposal. International Journal of Wildland Fire, 12(1), 23-37. https://doi.org/10.1071/WF02040

Santana, C. E., Jardel-Peláez, E., y Pere, P. (2009). Manejo del Fuego y Conservación de la Fauna Silvestre en ecosistemas forestales de montaña. En J. G. Flores Garnica (coord.), Impacto ambiental de incendios forestales (pp. 195-222). México: Editorial Mundi Prensa.

Skolmen, R. G., y Ledig, F. T. (1990). Eucalyptus globulus Labill. Bluegum eucalyptus. En R. Burns y B. Honkala (eds.), Silvics of North America, Hardwoods. Agric. Handbook 654 (pp. 299-304). Washington, DC: U.S. Department of Agriculture, Forest Service.

Tapias, R., Bertomeu, M., Gil, L., y Pardos, J. A. (1997). El papel evolutivo del fuego como factor de selección natural en masas de pinos mediterráneos. Estudio de los conos serotinos y la floración precoz. En F. Puertas Tricas y M. Rivas (eds.), Actas del I Congreso Forestal Hispano-Luso y II Congreso Forestal Español IRATI-97, V: (pp. 455-461). Pamplona: Gráficas Pamplona.

Terradas, J. (2001). Ecología de la vegetación. De la ecofisiología de las plantas a la dinámica de comunidades y paisajes. Barcelona, España: Ediciones Omega S.A.

Tirado Mejía, A. (1997). Colombia: siglo y medio de bipartidismo En J. O. Melo (coord.) Colombia Hoy. Perspectivas hacia el siglo XXI (pp. 103-175). Bogotá: Tercer Mundo.

Vasco, C. (1995). La teoría general de procesos y sistemas. Bogotá: Misión Ciencia Educación y Desarrollo. Colciencias.

Vélez, R. (2000). La defensa Contra incendios forestales fundamentos y experiencias. Madrid: McGraw Hill. 
Walker, B., Holling, C. S., Carpenter, S. R., y Kinzig, A. (2004). Resilience, adaptability and transformability in socialecological systems. Ecology and Society, 9(2), 5. Recuperado de http://www.ecologyandsociety.org/vol9/iss2/a $\mathrm{rt} 5$

\section{Notas}

* Artículo de investigación.

Este artículo es resultado del trabajo titulado Implementación de estrategias de manejo del fuego basado en Principios Ecológicos. El caso del Parque Ecológico Distrital de Montaña Entrenubes, Bogotá D. C. Fue realizado por Marcos Edwin Quiroga Palacio, en el 2017.

\section{Licencia Creative Commons CC BY 4.0}

Cómo citar este artículo: Quiroga Palacio, M. E., y Santiago Villa, H. M. (2019). Manejo del fuego como alternativa frente a los incendios forestales. El caso del Parque Entrenubes. Ambiente y Desarrollo, 23(45). https://doi.org/10.11144/Javeriana.ayd23-45.mfaf 